\title{
Axial $\mathrm{U}(1)$ symmetry and mesonic correlators at high temperature in $N_{f}=2$ lattice QCD
}

\author{
Kei Suzuki ${ }^{* a}$, Sinya Aoki ${ }^{b}$, Yasumichi Aoki ${ }^{c}$, Guido Cossu ${ }^{d}$, Hidenori Fukaya ${ }^{e}$, \\ Shoji Hashimoto ${ }^{f, g}$, Christian Rohrhofer ${ }^{e}$ (JLQCD Collaboration) \\ ${ }^{a}$ Advanced Science Research Center, Japan Atomic Energy Agency (JAEA), Tokai 319-1195, \\ Japan \\ ${ }^{b}$ Center for Gravitational Physics, Yukawa Institute for Theoretical Physics, Kyoto 606-8502, \\ Japan \\ ${ }^{c}$ RIKEN Center for Computational Science, Kobe 650-0047, Japan \\ ${ }^{d}$ School of Physics and Astronomy, The University of Edinburgh, Edinburgh EH9 3JZ, United \\ Kingdom \\ ${ }^{e}$ Department of Physics, Osaka University, Toyonaka 560-0043, Japan \\ ${ }^{f}$ KEK Theory Center, High Energy Accelerator Research Organization (KEK), Tsukuba \\ 305-0801, Japan \\ ${ }^{g}$ School of High Energy Accelerator Science, The Graduate University for Advanced Studies \\ (Sokendai), Tsukuba 305-0801, Japan
}

\begin{abstract}
We investigate the high-temperature phase of QCD using lattice QCD simulations with $N_{f}=2$ dynamical Möbius domain-wall fermions. On generated configurations, we study the axial $U(1)$ symmetry, overlap-Dirac spectra, screening masses from mesonic correlators, and topological susceptibility. We find that some of the observables are quite sensitive to lattice artifacts due to a small violation of the chiral symmetry. For those observables, we reweight the Möbius domainwall fermion determinant by that of the overlap fermion. We also check the volume dependence of observables. Our data near the chiral limit indicates a strong suppression of the axial $U(1)$ anomaly at temperatures $\geq 220 \mathrm{MeV}$.
\end{abstract}

37th International Symposium on Lattice Field Theory - Lattice2019

16-22 June 2019

Wuhan, China

* Speaker. 


\section{Introduction}

In the high-temperature region of quantum chromodynamics (QCD), one of open questions is the fate of the $U(1)_{A}$ symmetry. In the low-temperature phase, the $U(1)_{A}$ symmetry is known to be broken by a quantum anomaly which is related to topological excitations of gluon fields, e.g, instantons. In the high-temperature region with restored chiral symmetry (in other words, above the critical temperature, $T>T_{C}$ ), the restoration or violation of the $U(1)_{A}$ symmetry is still a longstanding problem not only in theoretical approaches $[1,2,3]$ but also in lattice QCD simulations at $N_{f}=2[4,5,6,7,8]$ and $N_{f}=2+1[9,10,11,12,13,14]$.

In older studies, lattice simulations reported a sizable $U(1)_{A}$ symmetry breaking above the critical temperature. However, many studies applied the staggered-type fermions, where chiral symmetry is explicitly broken, and it was difficult to precisely measure how much of $U(1)_{A}$ symmetry breaking is due to lattice artifacts. Recently, chiral fermions were employed to simulate lattice QCD at high temperature [4, 5, 7, 9, 10, 12, 13] (in Refs. [12, 13], only for valence quark sector). JLQCD Collaboration studied with $N_{f}=2$ chiral fermions [4, 7]. In Ref. [4], we generated the gauge ensembles with dynamical overlap fermions and applied a topology fixed approach at the $Q=0$ sector. In Ref. [7], gauge ensembles are generated with the Möbius domain-wall (MDW) fermions [15, 16], and a overlap/domain-wall reweighting technique [17, 7] was applied, where observables measured on MDW fermion ensembles are reweighted to those on overlap fermion ensembles. A disappearance of the $U(1)_{A}$ anomaly (at around $1.2 T_{C}$ ) was also reported in simulations with $N_{f}=2$ non-chiral fermions by other groups [6, 8]. In Ref. [14], they found that the $U(1)_{A}$ symmetry is good at $1.3 T_{c}$ but not near $T_{c}$.

In these proceedings, we report on our recent results of the observables at $T=220 \mathrm{MeV}$ such as the Dirac spectrum, $U(1)_{A}$ susceptibility, screening masses from mesonic correlators, and

Table 1: Numerical parameters of lattice simulations. $L^{3} \times L_{t}$ and $m$ are the lattice size and quark mass, respectively. $\bar{\Delta}_{\pi-\delta}^{\text {ov }}$ and $\chi_{t}$ are our results of the $U(1)_{A}$ susceptibility and topological susceptibility from the fermionic definition, respectively.

\begin{tabular}{cccc}
\hline \hline$L^{3} \times L_{t}$ & $a m$ & $\bar{\Delta}_{\pi-\delta}^{\text {ov }} a^{2}$ on OV & $\chi_{t} a^{4}$ \\
\hline $24^{3} \times 12$ & 0.001 & $1.5(0.6) \times 10^{-6}$ & $\approx 0$ \\
$24^{3} \times 12$ & 0.0025 & $3.6(1.3) \times 10^{-5}$ & $5.0(3.7) \times 10^{-8}$ \\
$24^{3} \times 12$ & 0.00375 & $0.00017(7)$ & $2.3(0.7) \times 10^{-7}$ \\
$24^{3} \times 12$ & 0.005 & $0.00091(42)$ & $9.0(2.0) \times 10^{-7}$ \\
$24^{3} \times 12$ & 0.01 & $0.00389(92)$ & $1.7(0.2) \times 10^{-6}$ \\
\hline $32^{3} \times 12$ & 0.001 & $1.8(1.4) \times 10^{-5}$ & $8.8(8.8) \times 10^{-12}$ \\
$32^{3} \times 12$ & 0.0025 & $0.00017(6)$ & $3.5(3.0) \times 10^{-8}$ \\
$32^{3} \times 12$ & 0.00375 & $0.00026(8)$ & $7.9(3.0) \times 10^{-8}$ \\
$32^{3} \times 12$ & 0.005 & $0.00291(188)$ & $9.3(1.9) \times 10^{-7}$ \\
$32^{3} \times 12$ & 0.01 & $0.01358(263)$ & $2.9(0.4) \times 10^{-6}$ \\
\hline $40^{3} \times 12$ & 0.005 & $0.00785(178)$ & $5.4(0.6) \times 10^{-7}$ \\
$40^{3} \times 12$ & 0.01 & $0.01162(140)$ & $2.0(0.2) \times 10^{-6}$ \\
\hline $48^{3} \times 12$ & 0.001 & $2.2(0.9) \times 10^{-6}$ & $4.2(4.3) \times 10^{-16}$ \\
$48^{3} \times 12$ & 0.0025 & $0.00012(4)$ & $4.9(4.4) \times 10^{-9}$ \\
$48^{3} \times 12$ & 0.00375 & $0.00032(12)$ & $1.5(0.7) \times 10^{-7}$ \\
$48^{3} \times 12$ & 0.005 & $0.00135(63)$ & $2.9(1.1) \times 10^{-7}$ \\
\hline \hline
\end{tabular}



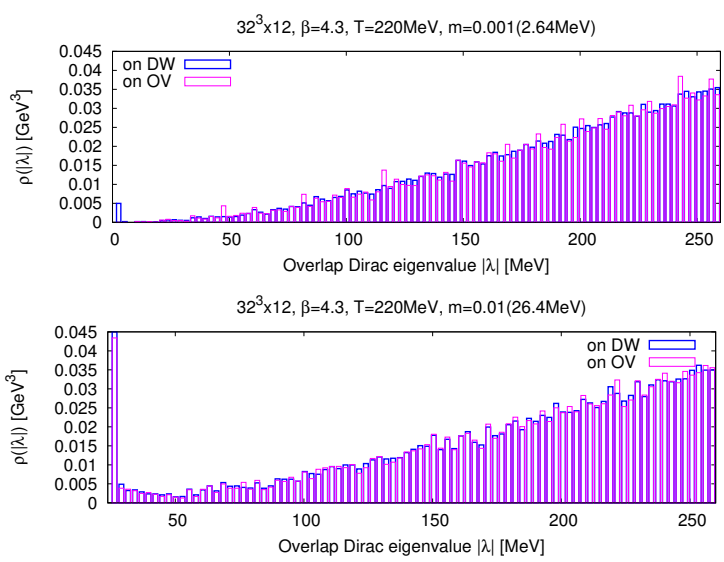

Figure 1: Spectral density $\rho(|\lambda|)$ for overlap-Dirac eigenvalues $\lambda$ at $T=220 \mathrm{MeV}$. Upper panel: $m=$ $2.64 \mathrm{MeV}$. Lower panel: $m=26.4 \mathrm{MeV}$.

topological susceptibility in $N_{f}=2$ lattice QCD simulations. The simulation parameters are summarized in Table 1. Our gauge ensembles are generated with the tree-level Symanzik improved gauge action and dynamical MDW fermions. We use the gauge coupling $\beta=4.30$ and the lattice spacing $1 / a=2.64 \mathrm{GeV}(a \sim 0.075 \mathrm{fm})$, which is finer than that of configurations used in the previous works $[4,7]$. We simulate lattice volumes $L=24,32,40,48$, and the length of the fifth dimension in the MDW fermion formulation is $L_{s}=16$. The physical quark mass (as the average of up and down quark masses) is estimated to be $a m=0.0014(2)$ (3.7(5) MeV). Some of our results were already reported in previous proceedings [18, 19, 20, 21].

\section{Overlap Dirac spectrum}

In Fig. 1, we plot spectral density of overlap Dirac eigenvalues, $\rho(\lambda)=(1 / V)\left\langle\sum_{\lambda^{\prime}} \delta\left(\lambda-\lambda^{\prime}\right)\right\rangle$ for two typical ensembles. The blue and magenta bins denote the spectra on the MDW fermions ensembles (DW) and reweighted overlap fermion ensembles (OV), respectively. At $m=2.64 \mathrm{MeV}$ for the OV ensembles, we find a suppression of both low eigenmodes and chiral zero modes. The suppression of the low eigenmodes is related to the $U(1)_{A}$ symmetry restoration in the light quark mass region. The disappearance of the chiral zero modes is related to the suppression of the topological susceptibility. At $m=26.4 \mathrm{MeV}$, low eigenmodes are enhanced, which is related to the $U(1)_{A}$ symmetry breaking.

\section{3. $U(1)_{A}$ susceptibility}

The $U(1)_{A}$ susceptibility $\Delta_{\pi-\delta}$ is an order parameter of the $U(1)_{A}$ symmetry breaking. This is defined from a spacetime integral of the difference between two-point correlators of isovectorpseudoscalar $\left(\pi^{a} \equiv i \bar{\psi} \tau^{a} \gamma_{5} \psi\right)$ and isovector-scalar $\left(\delta^{a} \equiv \bar{\psi} \tau^{a} \psi\right)$ operators:

$$
\Delta_{\pi-\delta} \equiv \chi_{\pi}-\chi_{\delta} \equiv \int d^{4} x\left\langle\pi^{a}(x) \pi^{a}(0)-\delta^{a}(x) \delta^{a}(0)\right\rangle,
$$

where $a$ is an isospin index in $N_{f}=2 \mathrm{QCD}$. The $U(1)_{A}$ susceptibility in the lattice theory is defined by a summation of low-lying eigenvalues of the overlap Dirac operator, $\lambda_{i}^{(\mathrm{ov}, m)}$ [22]:

$$
\Delta_{\pi-\delta}^{\mathrm{ov}}=\frac{1}{V\left(1-m^{2}\right)^{2}}\left\langle\sum_{i} \frac{2 m^{2}\left(1-\lambda_{i}^{(\mathrm{ov}, m) 2}\right)^{2}}{\lambda_{i}^{(\mathrm{ov}, m) 4}}\right\rangle,
$$




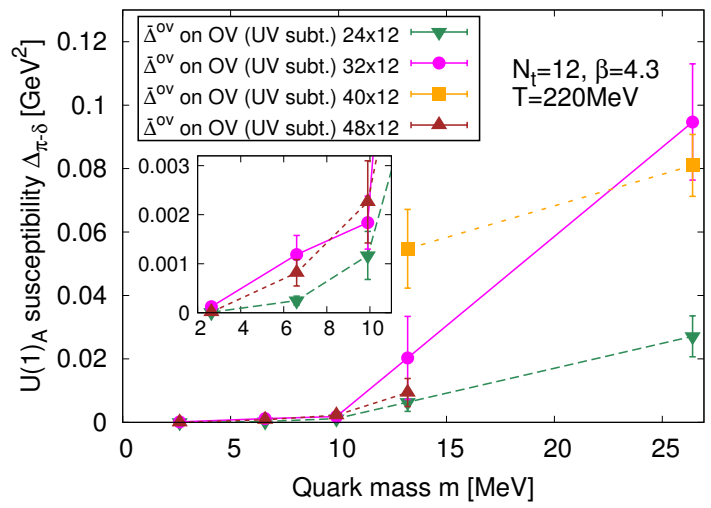

Figure 2: $U(1)_{A}$ susceptibilities, $\bar{\Delta}_{\pi-\delta}^{\text {ov }}$ (3.2), from the eigenvalue density of the overlap-Dirac operators at $T=220 \mathrm{MeV}$.

where we set the lattice spacing $a=1$. This summation is truncated at the lowest 40 eigenvalues. ${ }^{1}$

In Fig. 2, we show the $U(1)_{A}$ susceptibility at $T=220 \mathrm{MeV}$. In the light quark mass region, we find strong suppression of the $\Delta_{\pi-\delta}^{\text {ov }}$. For example, at the lowest quark mass and $L=32$, the ratio of $\Delta_{\pi-\delta}^{\text {ov }}$ to temperature is $\sqrt{\Delta_{\pi-\delta}^{o v}} / T \approx 5 \%$. The volume dependence is small for $L=24-48$. The data at different volumes are consistent except for the heaviest quark mass at $L=24$, whose aspect ratio against temperature is $L / L_{t}=2$.

\section{Screening mass difference from spatial mesonic correlators}

The screening mass is defined by the exponential decay of spatial correlators, which may be used to measure a violation of $U(1)_{A}$ symmetry. We investigate the difference between the effective screening masses

$$
\Delta m_{s c r}(z)=\left|m_{s c r}^{P S}(z)-m_{s c r}^{S}(z)\right|,
$$

where $m_{s c r}^{P S}(z)$ and $m_{s c r}^{S}(z)$ are the effective screening masses at a spatial coordinate $z$ for isovectorpseudoscalar $\left(\pi^{a} \equiv i \bar{\psi} \tau^{a} \gamma_{5} \psi\right)$ and isovector-scalar $\left(\delta^{a} \equiv \bar{\psi} \tau^{a} \psi\right)$ operators, respectively.

In Fig. 3, we show the difference between the effective screening masses measured by the MDW operator (without reweighting), where the horizontal axis is a dimensionless spatial distance $\left(z T=\left(n_{z} a / N_{t} a\right)=n_{z} / N_{t}\right)$. For the screening masses with light quark mass, we find a small value of $\Delta m_{s c r}(z T)$, which indicate the restoration of the $U(1)_{A}$ symmetry and it is consistent with the results of the $U(1)_{A}$ susceptibility $\bar{\Delta}_{\pi-\delta}^{\text {ov }}$. For heavy quark masses, the mass difference becomes large, which implies the $U(1)_{A}$ symmetry breaking.

\section{Topological susceptibility}

The topological susceptibility $\chi_{t}$ is defined as a gauge ensemble average of the topological charge $Q_{t}$ :

$$
\chi_{t}=\frac{\left\langle Q_{t}^{2}\right\rangle}{V},
$$

\footnotetext{
${ }^{1}$ From this definition, we further apply two types of subtractions: a subtraction of the contributions from chiral zero modes and an ultraviolet divergence (or lattice cutoff). For a justification of the zero mode subtraction, see Ref. [2, 7]. For the parametrization scheme of the lattice cutoff contribution by different valence quark masses, see Ref. [20, 21].
} 


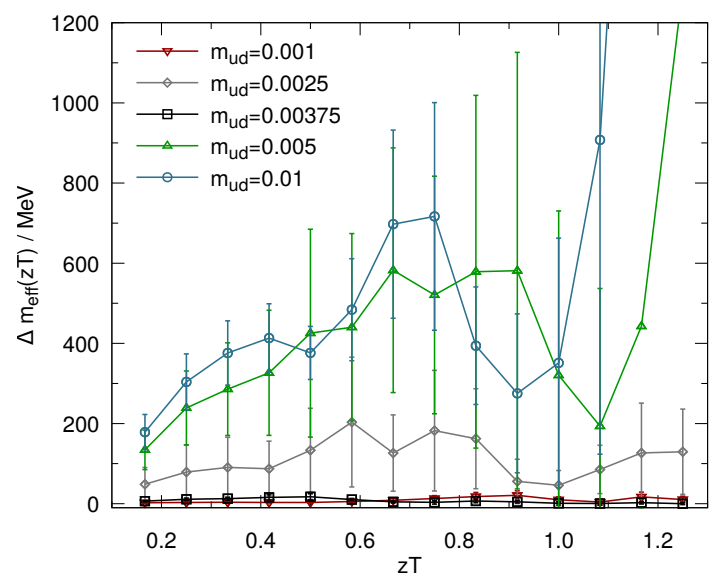

Figure 3: Difference between effective screening masses (4.1) from spatial mesonic collerators for $U(1)_{A}$ partners at $T=220 \mathrm{MeV}$ and $L=32$. The horizontal axis is defined as a dimensionless spatial distance $z T=\left(n_{z} a / N_{t} a\right)=n_{z} / N_{t}$.

For the topological charge $Q_{t}$, we employ two definitions. As a fermionic definition, $Q_{t}$ is defined through the index theorem for the overlap Dirac operator:

$$
Q_{t}=n_{+}-n_{-},
$$

where $n_{ \pm}$are the numbers of chiral zero modes with positive or negative chirality, respectively. As a gluonic definition, $Q_{t}$ is defined as a summation over spacetime $x$ at a flow time $t$ :

$$
Q_{t}(t)=\frac{1}{32 \pi^{2}} \sum_{x} \varepsilon^{\mu v \rho \sigma} \operatorname{Tr} F_{\mu v}(x, t) F_{\rho \sigma}(x, t),
$$

where $F_{\mu v}(x, t)$ is the clover-type discretization of the field strength tensor [23].2

In Fig. 4, we plot the topological susceptibility $\chi_{t}$ at $T=220 \mathrm{MeV}$. We show the results from the fermionic definition (5.2) on the OV ensembles and the gluonic definition (5.3) on the MDW ensembles, respectively. In the light quark mass region, $\chi_{t}$ is strongly suppressed with both the definitions. Furthermore, the volume dependence between $L=24$ and 48 is small. In the heavy quark mass region, the value of $\chi_{t}$ becomes nonzero, which is in agreement with the peak structure of the Dirac spectra in the lower panel of Fig. 1.

\section{Summary and discussion}

In these proceedings, we studied the high-temperature phase of QCD at $T=220 \mathrm{MeV}$ by using $N_{f}=2$ lattice QCD simulations with dynamical MDW fermions. We found small values of the $U(1)_{A}$ susceptibility (3.2) and the difference of mesonic screening masses (4.1) in light quark mass region, $m \lesssim 10 \mathrm{MeV}$, which indicates the $U(1)_{A}$ symmetry restoration in the chiral limit $(m \rightarrow 0)$. Furthermore, we found strong suppression of the topological susceptibility in the light-quark mass region. The mesonic and baryonic correlators at higher temperature were already reported in Refs. [24, 25, 26].

\footnotetext{
${ }^{2}$ This definition is usually not an integer, but we find a well-discretized distribution of $Q_{t}(t)$ at $t=5$.
} 


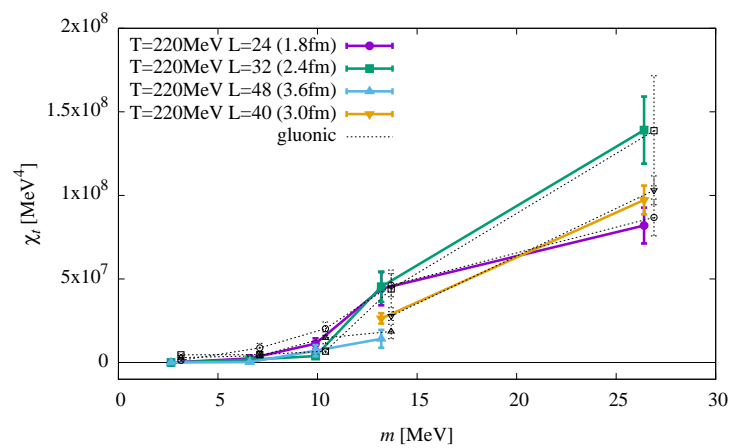

Figure 4: Topological susceptibilities $\chi_{t}$ at $T=220 \mathrm{MeV}$. Colored points: $\chi_{t}$ from the fermionic definition (5.2) on reweighted OV ensembles. Uncolored points: $\chi_{t}$ from the gluonic defnition (5.3) on MDW ensembles.

\section{Acknowledgment}

Numerical simulations are performed on IBM System Blue Gene Solution at KEK under a support of its Large Scale Simulation Program (No. 16/17-14) and Oakforest-PACS at JCAHPC under a support of the HPCI System Research Projects (Project IDs: hp170061, hp180061 and hp190090) and Multidisciplinary Cooperative Research Program in CCS, University of Tsukuba (Project IDs: xg17i032 and xg18i023). This work is supported in part by the Japanese Grant-inAid for Scientific Research (No. JP26247043, JP18H01216 and JP18H04484), and by MEXT as "Priority Issue on Post-K computer" (Elucidation of the Fundamental Laws and Evolution of the Universe) and by Joint Institute for Computational Fundamental Science (JICFuS).

\section{References}

[1] T. D. Cohen, $Q C D$ inequalities, the high temperature phase of $Q C D$, and $U(1)_{A}$ symmetry, Phys. Rev. D54 (1996) R1867 [hep-ph/ 9601216 ].

[2] S. Aoki, H. Fukaya and Y. Taniguchi, Chiral symmetry restoration, eigenvalue density of Dirac operator and axial U(1) anomaly at finite temperature, Phys. Rev. D86 (2012) 114512 [1209.2061].

[3] T. Kanazawa and N. Yamamoto, U (1) axial symmetry and Dirac spectra in QCD at high temperature, JHEP 01 (2016) 141 [1508. 02416 ].

[4] G. Cossu, S. Aoki, H. Fukaya, S. Hashimoto, T. Kaneko, H. Matsufuru et al., Finite temperature study of the axial U(1) symmetry on the lattice with overlap fermion formulation, Phys. Rev. D87 (2013) 114514 [1304.6145].

[5] TWQCD collaboration, T.-W. Chiu, W.-P. Chen, Y.-C. Chen, H.-Y. Chou and T.-H. Hsieh, Chiral symmetry and axial $U(1)$ symmetry in finite temperature $Q C D$ with domain-wall fermion, PoS LATTICE2013 (2014) 165 [1311.6220].

[6] B. B. Brandt, A. Francis, H. B. Meyer, O. Philipsen, D. Robaina and H. Wittig, On the strength of the $U_{A}(1)$ anomaly at the chiral phase transition in $N_{f}=2$ QCD, JHEP 12 (2016) 158 [1608.06882].

[7] JLQCD collaboration, A. Tomiya, G. Cossu, S. Aoki, H. Fukaya, S. Hashimoto, T. Kaneko et al., Evidence of effective axial U(1) symmetry restoration at high temperature QCD, Phys. Rev. D96 (2017) 034509 [1612.01908].

[8] K. I. Ishikawa, Y. Iwasaki, Y. Nakayama and T. Yoshie, Nature of chiral phase transition in two-flavor QCD, 1706.08872

[9] HotQCD collaboration, A. Bazavov et al., The chiral transition and $U(1)_{A}$ symmetry restoration from lattice QCD using Domain Wall Fermions, Phys. Rev. D86 (2012) 094503 [120 5 . 3535 ]. 
[10] LLNL/RBC collaboration, M. I. Buchoff et al., $Q C D$ chiral transition, $U(1)_{A}$ symmetry and the dirac spectrum using domain wall fermions, Phys. Rev. D89 (2014) 054514 [1309.4149].

[11] HотQCD collaboration, T. Bhattacharya et al., QCD Phase Transition with Chiral Quarks and Physical Quark Masses, Phys. Rev. Lett. 113 (2014) 082001 [1402.5175].

[12] V. Dick, F. Karsch, E. Laermann, S. Mukherjee and S. Sharma, Microscopic origin of $U_{A}(1)$ symmetry violation in the high temperature phase of QCD, Phys. Rev. D91 (2015) 094504 [1502.06190].

[13] L. Mazur, O. Kaczmarek, E. Laermann and S. Sharma, The fate of axial U(1) in 2+1 flavor QCD towards the chiral limit, PoS LATTICE2018 (2019) 153 [1811.08222].

[14] НотQCD collaboration, A. Bazavov et al., Meson screening masses in (2+1)-flavor QCD, Phys. Rev. D100 (2019) 094510 [1908.09552].

[15] R. C. Brower, H. Neff and K. Orginos, Möbius fermions, Nucl. Phys. Proc. Suppl. 153 (2006) 191 [hep-lat/0511031].

[16] R. C. Brower, H. Neff and K. Orginos, The Möbius domain wall fermion algorithm, Comput. Phys. Commun. 220 (2017) 1 [1206.5214].

[17] JLQCD collaboration, H. Fukaya, S. Aoki, G. Cossu, S. Hashimoto, T. Kaneko and J. Noaki, Overlap/Domain-wall reweighting, PoS LATTICE2013 (2014) 127 [1311. 4646].

[18] JLQCD collaboration, S. Aoki, Y. Aoki, G. Cossu, H. Fukaya, S. Hashimoto and K. Suzuki, Topological Susceptibility in $N_{f}=2$ QCD at Finite Temperature, EPJ Web Conf. 175 (2018) 07024 [1711.07537].

[19] JLQCD collaboration, K. Suzuki, S. Aoki, Y. Aoki, G. Cossu, H. Fukaya and S. Hashimoto, Axial U(1) symmetry at high temperature in 2-flavor lattice QCD, EPJ Web Conf. 175 (2018) 07025 [1711.09239].

[20] JLQCD collaboration, K. Suzuki, S. Aoki, Y. Aoki, G. Cossu, H. Fukaya and S. Hashimoto, Axial $U(1)$ symmetry and Dirac spectra in high-temperature phase of $N_{f}=2$ lattice $Q C D, P o S$ LATTICE2018 (2018) 152 [1812.06621].

[21] JLQCD collaboration, K. Suzuki, S. Aoki, Y. Aoki, G. Cossu, H. Fukaya and S. Hashimoto, Axial $U(1)$ symmetry, topology, and Dirac spectra at high temperature in $N_{f}=2$ lattice QCD, in 9th International Workshop on Chiral Dynamics (CD18) Durham, NC, USA, September 17-21, 2018, 2019, 1908.11684.

[22] JLQCD collaboration, G. Cossu, H. Fukaya, S. Hashimoto and A. Tomiya, Violation of chirality of the Möbius domain-wall Dirac operator from the eigenmodes, Phys. Rev. D93 (2016) 034507 [1510.07395].

[23] ALPHA collaboration, M. Bruno, S. Schaefer and R. Sommer, Topological susceptibility and the sampling of field space in $N_{f}=2$ lattice QCD simulations, JHEP 08 (2014) 150 [1406.5363].

[24] C. Rohrhofer, Y. Aoki, G. Cossu, H. Fukaya, L. Ya. Glozman, S. Hashimoto et al., Approximate degeneracy of $J=1$ spatial correlators in high temperature QCD, Phys. Rev. D96 (2017) 094501 [1707.01881].

[25] C. Rohrhofer, Y. Aoki, G. Cossu, H. Fukaya, C. Gattringer, L. Ya. Glozman et al., Symmetries of spatial meson correlators in high temperature QCD, Phys. Rev. D100 (2019) 014502 [1902.03191].

[26] C. Rohrhofer, Y. Aoki, G. Cossu, H. Fukaya, C. Gattringer, L. Ya. Glozman et al., Symmetries of the light hadron spectrum in high temperature QCD, in 37th International Symposium on Lattice Field Theory (Lattice 2019) Wuhan, Hubei, China, June 16-22, 2019, 2019, 1912.00678. 